\title{
Discussion of a Coupled Strength Attenuation Model for GFRP Composites in Hydrothermal Environments
}

\author{
Wei Chen, Yongxin Yang, and Biao Li \\ Central Research Institute of Building and Construction Co., Ltd., MCC Group, Beijing 100088, China \\ Correspondence should be addressed to Wei Chen; 923248809@qq.com
}

Received 24 June 2016; Revised 9 August 2016; Accepted 23 August 2016

Academic Editor: Jun Deng

Copyright ( 2016 Wei Chen et al. This is an open access article distributed under the Creative Commons Attribution License, which permits unrestricted use, distribution, and reproduction in any medium, provided the original work is properly cited.

\begin{abstract}
The existing attenuation models for the durability of FRP (fiber-reinforced polymer) composites in hydrothermal environments were compared, and a new coupled strength attenuation model with a temperature parameter was proposed in this paper. A series of durability experiments on GFRP sheets in hydrothermal environments were conducted to validate the accuracy and rationality of the new model. A comparison between experimental data and the calculation results of the coupled model indicated that the new model can fit better with the experimental data and effectively reflect the convergence phenomenon in the strength attenuation of GFRP in hydrothermal environments. With a temperature parameter included, the new model can better predict the service life of GFRP composites at different aging temperatures. According to the coupled attenuation model proposed in this paper, a concept and calculation method of the slow-aging time point are put forward, which can be convenient for the evaluation and design of GFRP structures with long-term durability.
\end{abstract}

\section{Introduction}

Research shows that FRP (fiber-reinforced polymer) composites possess great durability performance [1] compared with traditional civil engineering materials (i.e., steel and concrete), but some problems remain for further study. The performance degradation of FRPs in hydrothermal aging environments has aroused the attention of many scholars. Zhou [2] carried out experiments on pultruded GFRP square tubes in distilled water under temperatures of $20^{\circ} \mathrm{C}, 40^{\circ} \mathrm{C}$, and $60^{\circ} \mathrm{C}$. After 6 months of degradation, the results show that the mechanical properties of the GFRP samples decreased. The higher the temperature is, the greater the decrease in the mechanical properties is, and the faster the decrease rate is. Sun and $\mathrm{Li}$ [3] conducted experiments on FRPs in hydrothermal environments, and a new attenuation model for FRPs was obtained and corrected based on experimental data and Williams's model. Zhang et al. [4] analysed the performance degradation of FRPs in hydrothermal environments and put forward a parabolic-linear model. Yang et al. [5] conducted hydrothermal aging experiments on BFRP by changing the content of basalt fiber cloth, infiltration of resin and other factors, and the variations of the tensile strength, elongation ratio, and tensile elastic modulus were analysed. Berketis and Tzetzis [6] conducted experiments on GFRP plates in distilled water, and the results indicated that the prophase quality rising rate increases with the temperature, while the elastic modulus decreases first rapidly and then slowly. FRPs can very easily be subjected to hydrothermal aging. In response to the call for further understanding, many other scholars [7-9] have also conducted aging experiments to study the mechanical properties of FRPs in hydrothermal environments.

A few researchers have already proposed strength attenuation models of FRPs in hydrothermal environments. Williams et al. [10] proposed the WLF empirical equation (1) based on the time-temperature equivalence principle:

$$
\lg a_{T}=\lg \frac{t}{t_{s}}=\frac{-C_{1}\left(T-T_{s}\right)}{C_{2}+T-T_{s}},
$$

where $T_{s}$ and $t_{s}$ denote the reference temperature and time, respectively, and $C_{1}$ and $C_{2}$ are empirical constants. The strength of FRPs under different temperatures could be translated into the strength under the same temperature based on the time-temperature equivalence principle. The 
relationship between the residual strength and aging time can be presented as

$$
P_{t}=\frac{P_{0}}{100}[a \ln (t)+b],
$$

where $P_{t}$ denotes the residual strength, $P_{0}$ denotes the initial strength, and $a$ and $b$ are undetermined constants.

Gunyaev et al. [11] established a semiempirical mathematical model for the median aging residual strength. The aging process of FRPs could be regarded as irreversible, and the strength attenuation model can be expressed as

$$
S=S_{0}+\eta\left(1-e^{-\lambda}\right)-\beta \ln (1+\theta t),
$$

where $S$ denotes the strength after aging time $(t), S_{0}$ denotes the initial strength, $\eta$ and $\beta$ are material parameters, and $\lambda$ and $\theta$ are environmental parameters.

Guo [12] analysed the mechanical properties of FRPs in a hydrothermal environment and found that the mechanical properties first decrease rapidly and then slowly or even not at all. Based on this phenomenon, a bilinear attenuation model was proposed. Zhang et al. [3] improved the model by including the nonlinear law of the degradation of FRPs, such that the parabolic-linear attenuation model was proposed.

A comparison of the existing attenuation models indicates that Williams's model fits the experimental data well, but there is some error in the data of the later aging process. It is widely confirmed that Gunyaev et al.s model fits better with the experimental data, while the complexity caused by a large number of parameters in the model makes it inconvenient to use. Guo bilinear model is easy to calculate, but it cannot reflect the nonlinear law of the performance degradation of FRPs. Zhang parabolic-linear model has a good prediction capability at early aging times, but the second stage of the linear part of the model cannot reflect the gradual convergence of the degradation. In addition, none of the models above contain a temperature parameter. Williams's model has to translate data of the strength reduction under different temperatures into that of the same temperature and then calculate a single-factor model that is only related to the aging time factor. The other three models lack consideration of the influence of temperature, as the parameters are different for the same material at different temperatures, making it inconvenient to predict the strength attenuation of FRPs under conditions of a specific temperature. Therefore, a more convenient and reasonable model is urgently needed. This paper proposes a new model for the strength attenuation of GFRP containing a temperature parameter. The parameters of the model remain unchanged for a single material or product. The coupled model was proposed based on previous studies and a mechanism analysis. A series of durability experiments under hydrothermal environments were conducted to verify the accuracy of the proposed model.

\section{Single-Factor Model Analysis}

2.1. Temperature-Factor Model. According to the Arrhenius formula, the relationship between the strength reduction

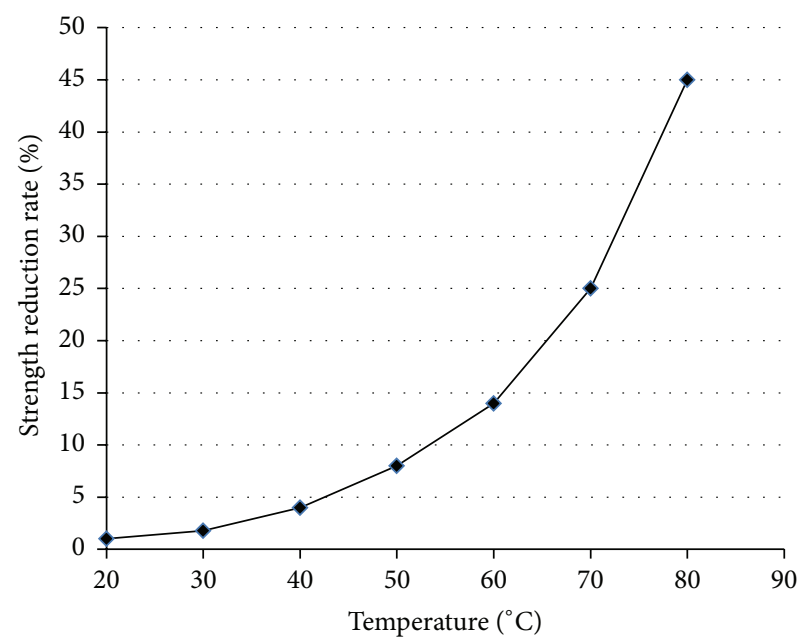

FIGURE 1: Relationship between strength reduction rate and temperature.

and temperature presents as the higher the temperature, the greater the strength reduction (within a certain temperature range). The slope of the tangent of the strength reduction curve as a function of temperature is gradually increasing. This indicates that the relationship between the strength reduction and temperature may be exponential or multinomial. Many scholars [13-17] reached the same conclusion in their research. It is widely believed that the increase in temperature can accelerate the aging of the GFRPs. Taking the natural index part of the Arrhenius formula into consideration, it is appropriate to assume that the strength reduction presents a natural exponential growth relationship with the temperature rising and that it can approximately simulate the influence of the temperature in the aging of FRPs. Robert and Fam [18] obtained experimental data from aging experiments of GFRP bars in a hydrothermal environment to support this opinion, as shown in Figure 1, from which it could be observed that the strength reduction shows an approximately exponential growth relationship with the temperature.

Therefore, assume that the strength reduction shows a natural exponential growth relationship with the temperature, and the attenuation model of the temperature can be expressed as

$$
D=A \cdot e^{B T},
$$

where $D$ denotes the strength reduction, $T$ denotes the temperature, and $A$ and $B$ are undetermined constants related to the time and temperature, respectively.

According to (4), if the aging temperature increases from $T_{0}$ to $T$, the strength reduction should be multiplied by influence factor $\lambda_{\Delta T}$, whereas $\lambda_{\Delta T}=D_{T} / D_{T_{0}}=e^{B\left(T-T_{0}\right)}$. In this model, $B$ can be considered as a constant for the same material, and all the time-dependent effects are included in parameter $A$. For certain materials and conditions, influence factor $\lambda_{\Delta T}$ is only affected by temperature difference $\Delta T$.

To verify the accuracy of the model, a series of durability experiments on GFRP sheets under different temperatures was conducted. The specimens for the tests were made of 


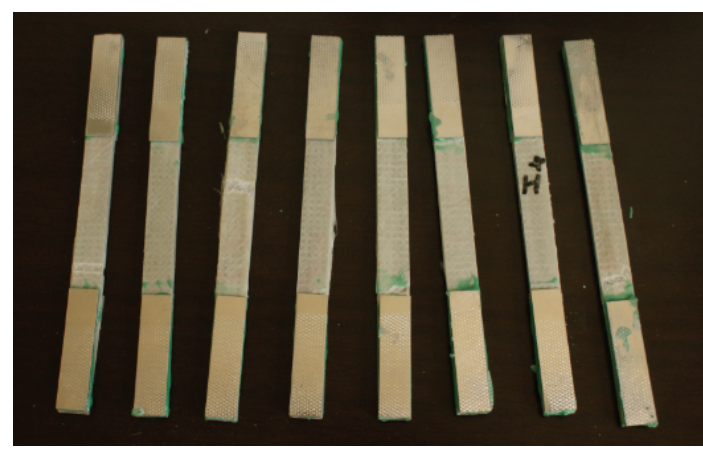

(a)

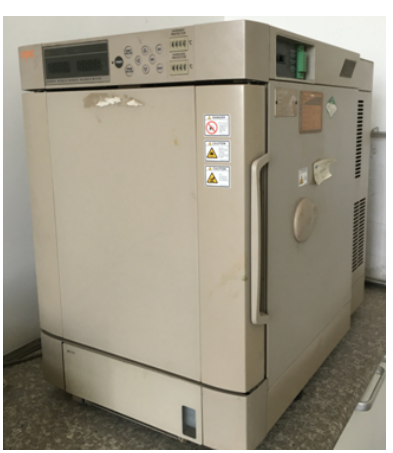

(b)

FIGURE 2: Experimental pictures: (a) samples of GFRP sheet and (b) the hydrothermal aging box.

TABLE 1: Strength of the samples after aging tests (MPa).

\begin{tabular}{lccccc}
\hline Tem. & \multicolumn{5}{c}{ Time } \\
& $42 \mathrm{~d}$ & $83 \mathrm{~d}$ & $124 \mathrm{~d}$ & $185 \mathrm{~d}$ & $250 \mathrm{~d}$ \\
\hline $25^{\circ} \mathrm{C}$ & 982 & 960 & 942 & 927 & 922 \\
$50^{\circ} \mathrm{C}$ & 1034 & 940 & 922 & 895 & 898 \\
$75^{\circ} \mathrm{C}$ & 958 & 912 & 890 & 856 & 854 \\
\hline
\end{tabular}

TABLE 2: Strength reduction rates of the samples (\%).

\begin{tabular}{lccccc}
\hline Tem. & & \multicolumn{5}{c}{ Time } \\
& $42 \mathrm{~d}$ & $83 \mathrm{~d}$ & $124 \mathrm{~d}$ & $185 \mathrm{~d}$ & $250 \mathrm{~d}$ \\
\hline $25^{\circ} \mathrm{C}$ & 2.87 & 5.06 & 6.82 & 8.31 & 8.51 \\
$50^{\circ} \mathrm{C}$ & -2.28 & 7.02 & 8.80 & 11.47 & 11.18 \\
$75^{\circ} \mathrm{C}$ & 5.12 & 9.79 & 11.97 & 15.33 & 15.53 \\
\hline
\end{tabular}

glass fiber fabric embedded in epoxy matrix and then cured for $24 \mathrm{~h}$ in laboratory environment (a temperature of $23^{\circ} \mathrm{C}$ and a humidity of 50\%) before the experiments. The aging temperatures of the experiments were set to $25^{\circ} \mathrm{C}, 50^{\circ} \mathrm{C}$, and $75^{\circ} \mathrm{C}$, and the environmental humidity was $95 \%$. Figure 2 shows the samples of the GFRP sheets and the hydrothermal aging box that was used.

The average strength of the GFRP sheets before the aging tests (after curing for $24 \mathrm{~h}$ ) is $1011 \mathrm{MPa}$. The strengths of the samples were tested after different aging times. The results of the tensile tests after aging are summarized in Table 1.

Based on the original data in Table 1, the strength reduction rates were calculated and are shown in Table 2.

It can be observed from Table 2 that after being exposed to a hydrothermal environment for 83 days, the strength reduction rates were $5.06 \%, 7.02 \%$, and $9.79 \%$ at $25^{\circ} \mathrm{C}$, $50^{\circ} \mathrm{C}$, and $75^{\circ} \mathrm{C}$, respectively. From the theoretical analysis of the new temperature model, the influence factor can be calculated as $\lambda_{\Delta T}=D_{T} / D_{T_{0}}=e^{B \Delta T}$. Combined with the strength reduction results above, the influence factor can be obtained as follows: $\lambda_{25}^{1}=D_{50} / D_{25}=7.02 / 5.06=$ 1.387 and $\lambda_{25}^{2}=D_{75} / D_{50}=9.79 / 7.02=1.395$. The two numbers are very close, which also reflects the reliability of the temperature attenuation model proposed by the authors. As for the data after 124 days, 185 days, and 250 days of aging, the values of influence factor are calculated as $\lambda_{25}^{1}=1.290$, 1.381 , and 1.314, respectively, and $\lambda_{25}^{2}=1.359,1.336$, and 1.38 , respectively. The values of the influence factor are close to those calculated after 83 days of aging, which proves the hypothesis that the influence factor and parameter $B$ in the new model as well as the shape of the temperature model that is mainly determined by parameter $B$ will not change with the change in the aging time. When the influence factor of the experiments is calculated, the data after 42 days of aging were ignored as abnormal data, which may be due to the second curing (i.e., postcuring) process which occurred in the specimens, and the average value of the influence factors can be obtained as $\bar{\lambda}_{25}=e^{B \Delta T}=e^{25 B}=1.357$, from which parameter $B$ for the material used in the experiments can be obtained as $B=0.012$. It must be clarified that, for different materials or different products, although the formula of the model is still applicable, the parameters in the model will change and need to be recalculated.

2.2. Aging Time-Factor Model. Extensive research has been conducted to study the attenuation law of strength for the GFRPs. Most of the studies [3,13,19-21] present the result that the strength of the GFRPs decreases rapidly at the early aging time and then goes slower and slower. To correspond with the natural index in the temperature model, a new model of aging for the time factor of GFRPs was proposed: $D=$ $e^{-C / t} . t$ denotes the aging time and $C$ is a constant number for certain materials. It can be observed that in this model, $D$ tends to be 0 when $t$ tends to be 0 , and $D$ tends to be 1 when $t$ tends to be infinity. Some FRP materials present an early convergence phenomenon, and the strength reduction cannot reach 1 . Based on this situation, parameter $M$ was introduced, and the new aging model can be represented by the following expression:

$$
D=M e^{-C / t} \quad(0<M<1) .
$$

The new aging model can intuitively show the relationship between the strength reduction and the aging time, and it is more accurate than the linear model.

The experimental data under the temperature of $50^{\circ} \mathrm{C}$ (Table 2) were used to validate the accuracy of the model. 


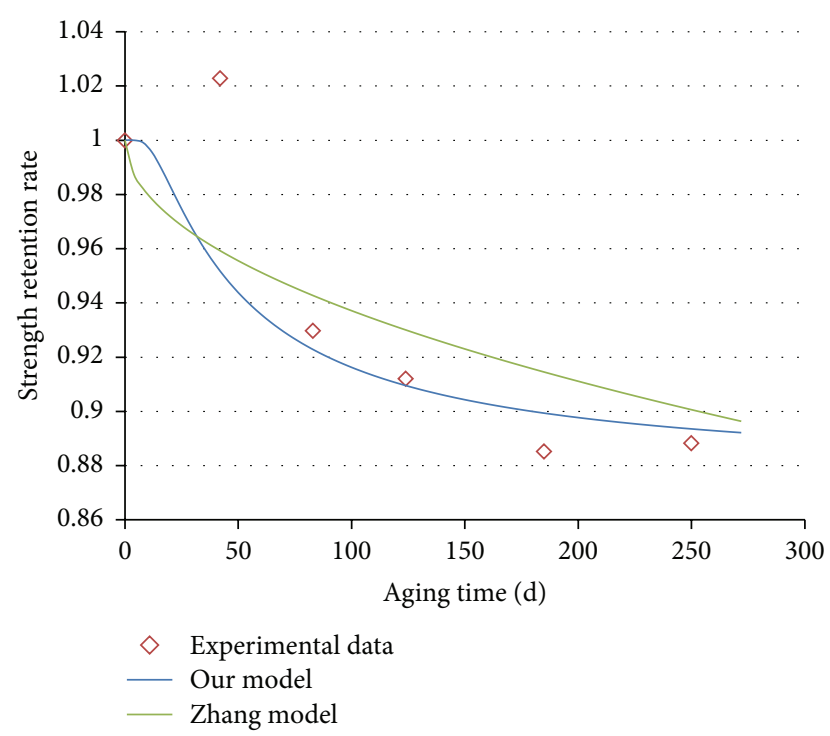

FIGURE 3: Comparison of models and experimental data at $50^{\circ} \mathrm{C}$.

Through numerical fitting with (5), the values of the parameters were obtained: $M=0.125$ and $C=40$. Then, the strength attenuation model for the material used in the experiments is $D=0.125 e^{-40 / t}$. This new model is presented in Figure 3 and compared with Zhang parabolic-linear model.

Generally speaking, the strength of the GFRPs decreases rapidly at first and then slowly, and the aging rate becomes slower and slower over the aging time with a trend of convergence. It could be learned from the experimental data that, at a condition of $50^{\circ} \mathrm{C}$, the decrease of the strength began to slow down after exposure to a hydrothermal environment for 185 days, which indicates that the aging model proposed in this paper can better reflect this convergence phenomenon and can better fit the experimental data.

\section{Coupled Model Analysis}

From the analysis of the single-factor model, the relationship between the strength reduction and temperature could be expressed by (4). In this equation, $A$ is a parameter related to the influence of aging time. Additionally, the influence of the aging time was analysed and a new model for the aging time factor was obtained (5). When considering the influences of both the temperature and aging time, parameter $A$ in (4) could be replaced by the aging time factor model (5). After mathematical simplification, the following coupled model has been obtained:

$$
D=N \cdot e^{B T-C / t}
$$

where $N$ is a material parameter $(0<N<1)$, and $B$ and $C$ are undetermined constants related to the temperature and time, respectively.

Equation (6) is the proposed coupled model of strength attenuation with a temperature parameter for GFRP composites. It shows that the strength reduction rate increases with the rise of temperature and the aging time. The sensitivity to
TABLE 3: Values of material parameter $N$.

\begin{tabular}{lccccc}
\hline Tem. & & \multicolumn{5}{c}{ Time } \\
& $42 \mathrm{~d}$ & $83 \mathrm{~d}$ & $124 \mathrm{~d}$ & $185 \mathrm{~d}$ & $250 \mathrm{~d}$ \\
\hline $25^{\circ} \mathrm{C}$ & 0.054 & 0.063 & 0.070 & 0.076 & 0.074 \\
$50^{\circ} \mathrm{C}$ & -0.032 & 0.062 & 0.067 & 0.078 & 0.072 \\
$75^{\circ} \mathrm{C}$ & 0.053 & 0.064 & 0.067 & 0.077 & 0.074 \\
\hline
\end{tabular}

these two factors can also be obtained. A larger value of $B$ indicates a greater effect of the temperature, whereas a larger value of $C$ represents a slower convergence of the material in the process of aging. The values of $B$ and $N$ can determine the approach limit value of the strength reduction under the assumption of the strength attenuation model: $D_{\max }=N$. $e^{B T}$.

For the material used in our experiments, the value of parameter $B$ was obtained in the analysis of the temperature model above $(B=0.012)$, and the value of parameter $C$ was obtained in the analysis of the aging time model $(C=40)$. According to (6), the coupled model for our experiments can be expressed by the following equation:

$$
D=N e^{0.014 T-40 / t} \text {. }
$$

There is only one undetermined parameter, $N$, in (7). The experimental data (shown in Table 2) of strength reduction rate under different aging times $(t)$ and temperatures $(T)$ were used to calculate the values of parameter $N$, which are shown in Table 3.

According to the results shown in Table 3 , it is obvious that the values of $N$ are close to each other (except for the abnormal data after 42 days of aging, which has been explained in the temperature-factor model), reflecting the feasibility and accuracy of the theoretical model proposed in this paper. The average value of $N$ can be calculated as $\bar{N}=0.070$. And (7) could be expressed by the following equation:

$$
D=0.070 \cdot e^{0.012 \cdot T-40 / t} .
$$

It must be clarified that the parameters in (8) are only appropriate for the condition of our experiments. The experiments were conducted only as evidence to validate the accuracy and rationality of the model, and a specific model for the experiments (8) was used to present the superiority of the new proposed model. For other materials or products, although the formula of the model (6) is still applicable, the parameters of the model will change and need to be recalculated. According to (8), it will take 270 days for the strength to decrease by $11 \%$ at $50^{\circ} \mathrm{C}$, whereas, in an $80^{\circ} \mathrm{C}$ environment, it will only take 80 days to reach the same strength reduction. The new coupled model can conveniently predict the durability of FRP materials under different temperatures. A strength reduction curve as a function of the aging time has been put forward based on the new coupled model (Figure 4) for the material used in our experiments. After comparison with the experimental data, it could be noted that despite the abnormal data after aging for 42 days, the experimental data can basically fit the coupled model proposed by the paper. 


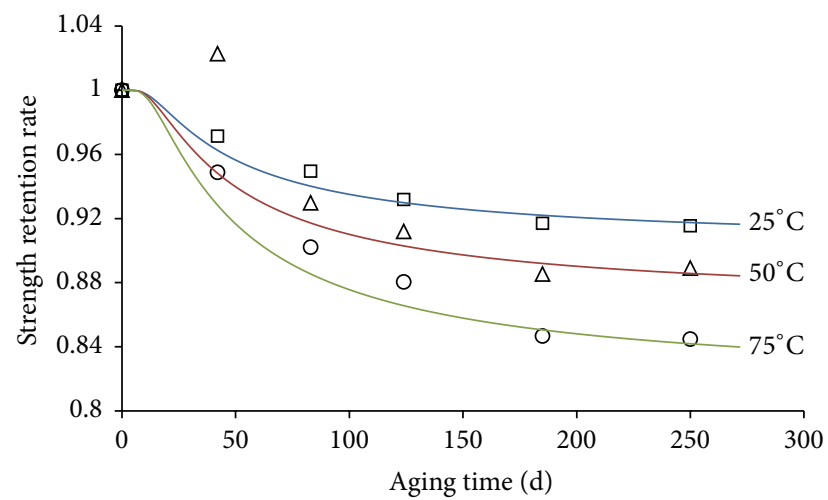

Figure 4: Strength decline rates under different temperatures.

From the aging experimental data and the strength attenuation curve given in Figure 4, it could be found that after aging for a certain time, the reduction rate slowed down and the strength gradually stabilized. Some scholars [22] believe that the product of the early reaction of aging in GFRPs plays a protective role in later reactions, such that the aging rate slows down. This convergence phenomenon could be well presented in the new coupled model (6). The aging time when the strength reduction reaches $90 \%$ of the approach limit reduction could be regarded as the transition point, after which the strength of the GFRPs could be regarded as stable. This transition point could be expressed as a slowaging time point $t_{i}$. According to (6), $D_{\max }=N \cdot e^{B T}$, and $D_{90 \%}=N \cdot e^{B T-C / t_{i}}$. If the conception of $t_{i}$ indicates that $D_{\max } / D_{90 \%}=90 \%$, then $t_{i}$ could be calculated as the formula shown in (9). It is obvious that the value of $t_{i}$ is only related to time parameter $C$. This is reasonable because, in the coupled model, the value of parameter $C$ determines the variation trend of the strength reduction related to the aging time. For instance, the value of $C$ has already been obtained $(C=40)$ for the material used in the experiments of this paper, so that the value of the slow-aging time point could be calculated as $t_{i}=379.7 d$, which means that, after 379.7 days of aging, the strength reduction had reached $90 \%$ of the approach limit reduction for the material used in the exposure environment. In practical engineering, if the strength of FRPs at $t_{i}$ could be obtained, it can roughly represent the long-term strength of the material. Furthermore, if the strength of the material at $t_{i}$ can meet the design requirements, it could be interpreted that the structure can meet the long-term service requirements. This can provide a reference for the evaluation and design of the long-term stability of FRP structures. It is important to note that for some FRP materials under certain conditions, the strength reduction rate does not slow down and there is no limit for strength reduction, so that the slow-aging time point becomes meaningless under this situation:

$$
t_{i}=\frac{C}{\ln 10-2 \ln 3} .
$$

In conclusion, not only can the new model in (6) be applied to analyse and predict the strength reduction of GFRP with a time factor, but it can also intuitively indicate the effect of temperature, and its accuracy and rationality were verified by experimental data. It should be noted that, for different materials or products, the parameters in the equation will change and should be calculated by experiments. The model also provides a certain reference value for other materials (e.g., CFRP and BFRP) and corrosive environments (e.g., acid, alkali, and salt chemical corrosion solutions), but its accuracy remains for further study.

\section{Conclusions}

The paper proposes a coupled model of strength attenuation for GFRP composites in hydrothermal environments. Although the aging mechanism has not yet been confirmed, single-factor models of the temperature and aging time were proposed based on the existing research results, and the coupled model was then obtained. A series of durability experiments on GFRP sheets in hydrothermal environments were conducted to validate the accuracy and rationality of the model. On the basis of the coupled model, the concept and calculation method of the slow-aging time point were presented. The following conclusions can be drawn from this study:

(1) A temperature-factor model was proposed based on the Arrhenius formula and existing studies, and it indicated that the strength reduction rate shows a natural exponential growth relationship with the temperature. The accuracy and rationality of the temperature were verified by the results of durability experiments, based on which the calculated values of influence factor $\lambda_{\Delta T}$ are close after different aging times.

(2) A new strength attenuation model with aging time factor was proposed based on the data of previous studies. Experimental data were used to support the new model, and it was shown that the new model can fit the experimental data better than the paraboliclinear model. The new model can also reflect the convergence phenomenon found in the experimental data.

(3) A coupled model for the strength attenuation of GFRP was obtained based on the single-factor models. According to the coupled model, the degradation curves of the strength of GFRP under $25^{\circ} \mathrm{C}, 50^{\circ} \mathrm{C}$, and $75^{\circ} \mathrm{C}$ were obtained. Despite the abnormal data after 42 days of aging, the experimental data can basically fit the coupled model proposed by the paper.

(4) According to the coupled model, the concept and calculation method of the slow-aging time point $t_{i}$ were proposed, which can provide a reference for the evaluation and design of the long-term stability of FRP structures.

\section{Competing Interests}

The authors declare that there are no competing interests regarding the publication of this paper. 


\section{Acknowledgments}

The authors gratefully acknowledge the financial support provided by the China 973 Program (Project no. 2012CB026205) and the Project of Water Conservancy Scientific Research and Technical Extension in Shandong Province (Project no. SDSLKY201407).

\section{References}

[1] Q. R. Yue and Y. X. Yang, "Introduction to durability of concrete strengthened with fiber reinforced polymers," Journal of Building Structures, vol. 30, no. 6, pp. 8-15, 2009.

[2] M. Zhou, Study on the hydrothermal durability of GFRP and BFRP composites [Ph.D. thesis], Harbin Institute of Technology, Harbin, China, 2013.

[3] B. Sun and Y. Li, "Study on the aging behavior of FRP materials and the prediction of its durability," Fiber Reinforced Plastics/Composites, vol. 4, pp. 29-35, 2013.

[4] Y. H. Zhang, Y. X. Yang, Y. Yao et al., "The durability test and attenuation unified model of FRP performance at hot and humid environment," Industrial Construction, vol. 10, pp. 46-50, 2014.

[5] Y. X. Yang, M. Yang, Y. Zhao et al., "Experimental study on durability of BFRP," Industrial Construction, vol. 6, pp. 11-13, 2007.

[6] K. Berketis and D. Tzetzis, "The compression-after-impact strength of woven and non-crimp fabric reinforced composites subjected to long-term water immersion ageing," Journal of Materials Science, vol. 45, no. 20, pp. 5611-5623, 2010.

[7] H. Rahimi, "Environmental durability of materials and bonded joints involving fiber reinforced polymer and concrete," in Proceedings of the 1st Conference on Application of FRP Composites in Construction and Rehabilitation of Structures, pp. 76-80, 2004.

[8] G. Xian, H. Li, and X. Su, "Effects of immersion and sustained bending on water absorption and thermomechanical properties of ultraviolet cured glass fiber-reinforced acylate polymer composites," Journal of Composite Materials, vol. 47, no. 18, pp. 22752285, 2013.

[9] A. R. Hutchinson and L. C. Hollaway, Environmental Durability Strengthening Concrete Structures with Bonding Fiber-Reinforced Composites, Wood Head Publishing, 1999.

[10] M. J. He, W. X. Chen, and X. X. Dong, Polymer Physics, Fudan University Press, Shanghai, China, 2000.

[11] G. M. Gunyaev, E. N. Kablov, and V. M. Aleksashin, "Modification of construction carbon-reinforced plastics with carbon nanoparticles," Russian Journal of General Chemistry, vol. 81, no. 5, pp. 970-977, 2011.

[12] C. H. Guo, Evaluation study on FRP mechanical properties and durability of FRP reinforced concrete [Ph.D. thesis], Central Research Institute of building and Construction CO. LTD, MCC Group, 2006.

[13] S. Li, H. T. Ren, C. K. Huang, and M. H. Shi, "Durability research of FRP sheet under temperature and alkali factor," Journal of Building Structures, no. 1, pp. 94-99, 2010.

[14] Z.-S. Guo, J. Feng, H. Wang, H. Hu, and J. Zhang, "A new temperature-dependent modulus model of glass/epoxy composite at elevated temperatures," Journal of Composite Materials, vol. 47, no. 26, pp. 3303-3310, 2013.
[15] I. Nishizaki and S. Meiarashi, "Long-term deterioration of GFRP in water and moist environment," Journal of Composites for Construction, vol. 6, no. 1, pp. 21-27, 2002.

[16] A. S. M. Kamal and M. Boulfiza, "Durability of GFRP rebars in simulated concrete solutions under accelerated aging conditions," Journal of Composites for Construction, vol. 15, no. 4, pp. 473-481, 2011.

[17] F. Ellyin and C. Rohrbacher, "Effect of aqueous environment and temperature on glass-fibre epoxy resin composites," Journal of Reinforced Plastics and Composites, vol. 19, no. 17, pp. 1405$1427,2000$.

[18] M. Robert and A. Fam, "Long-term performance of GFRP tubes filled with concrete and subjected to salt solution," Journal of Composites for Construction, vol. 16, no. 2, pp. 217-224, 2012.

[19] W. C. Xue and K. Fu, "Experimental research on mechanical behaviors of GFRP bridge decks under alkaline solution," Journal of Reinforced Plastics and Composites, vol. 32, no. 23, pp. 1835-1841, 2013.

[20] X. Jiang, H. Kolstein, F. Bijlaard, and X. Qiang, "Effects of hygrothermal aging on glass-fibre reinforced polymer laminates and adhesive of FRP composite bridge: moisture diffusion characteristics," Composites Part A: Applied Science and Manufacturing, vol. 57, pp. 49-58, 2014.

[21] H.-C. Wu and A. Yan, "Durability simulation of FRP bridge decks subject to weathering," Composites Part B: Engineering, vol. 51, pp. 162-168, 2013.

[22] M. Kotani, Y. Yasufuku, Y. Tamaishi, and H. Kawada, "Study of strength degradation mechanism of woven GFRP in water environment," Journal of Solid Mechanics and Materials Engineering, vol. 4, no. 11, pp. 1574-1584, 2010. 

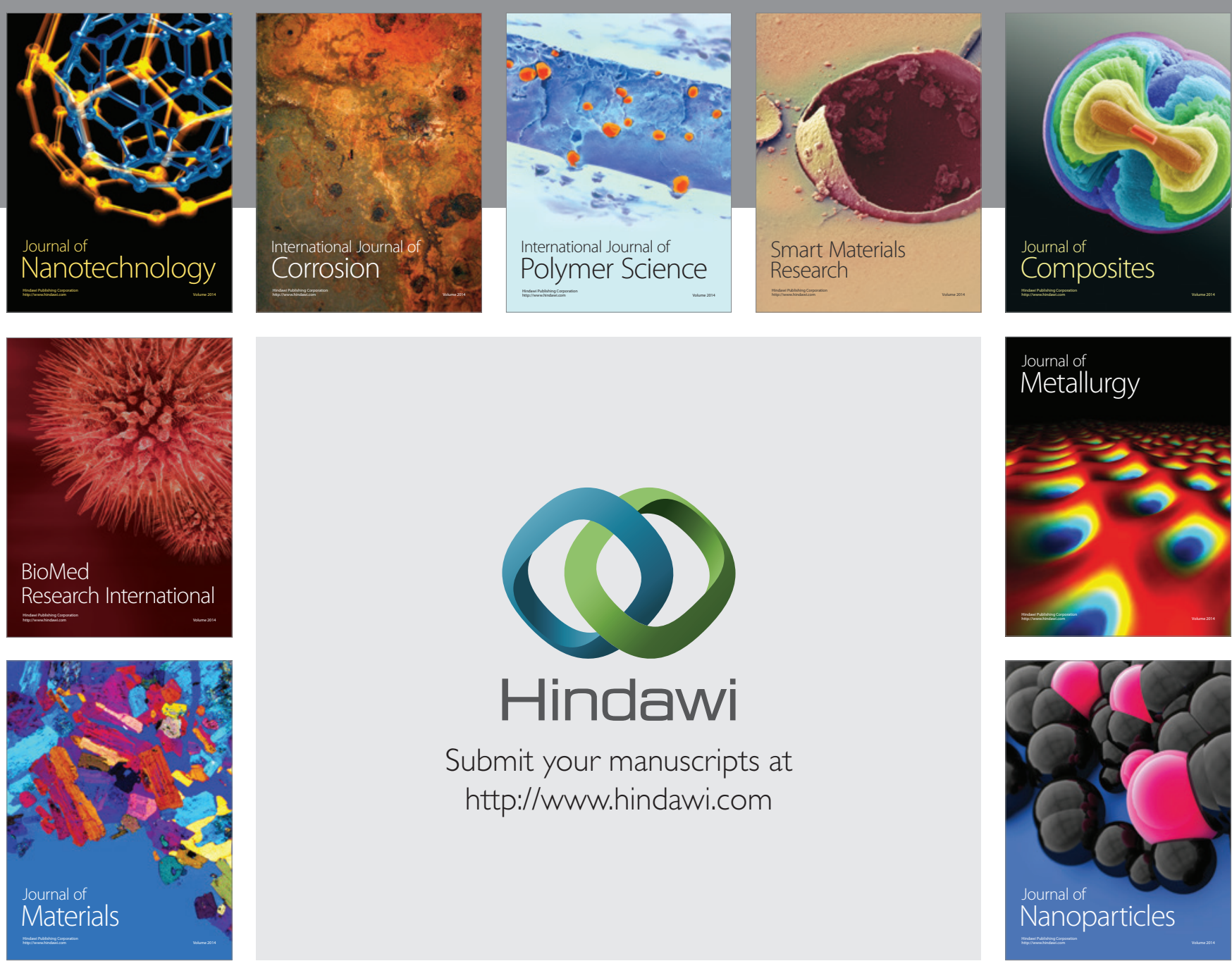

\section{Hindawi}

Submit your manuscripts at

http://www.hindawi.com

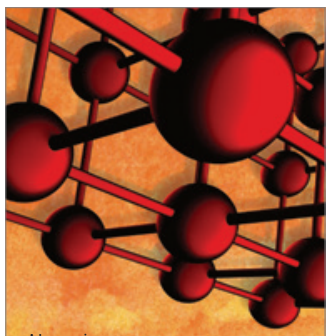

Materials Science and Engineering
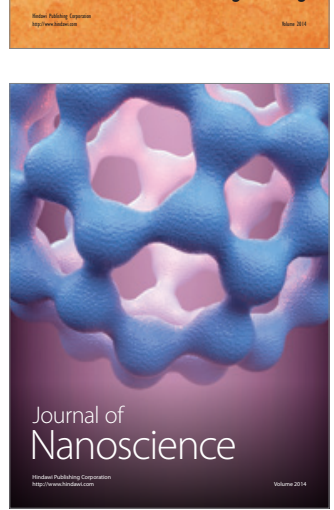
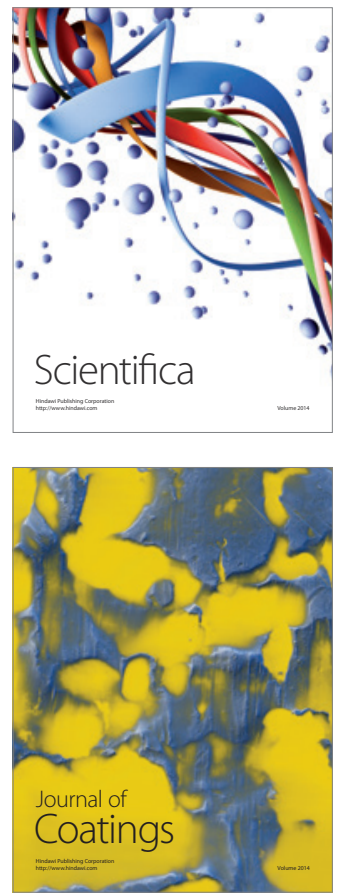
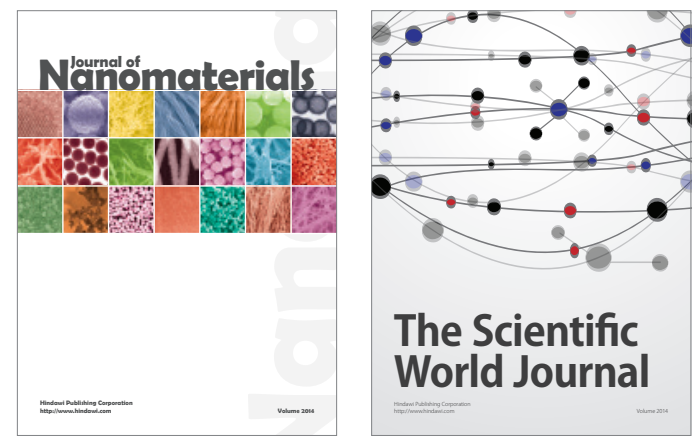

The Scientific World Journal
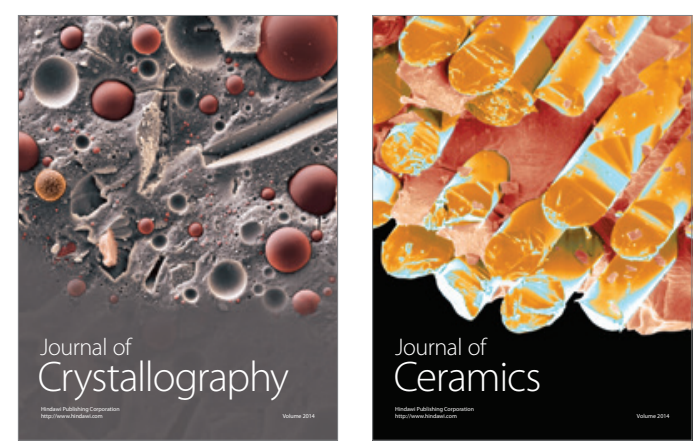
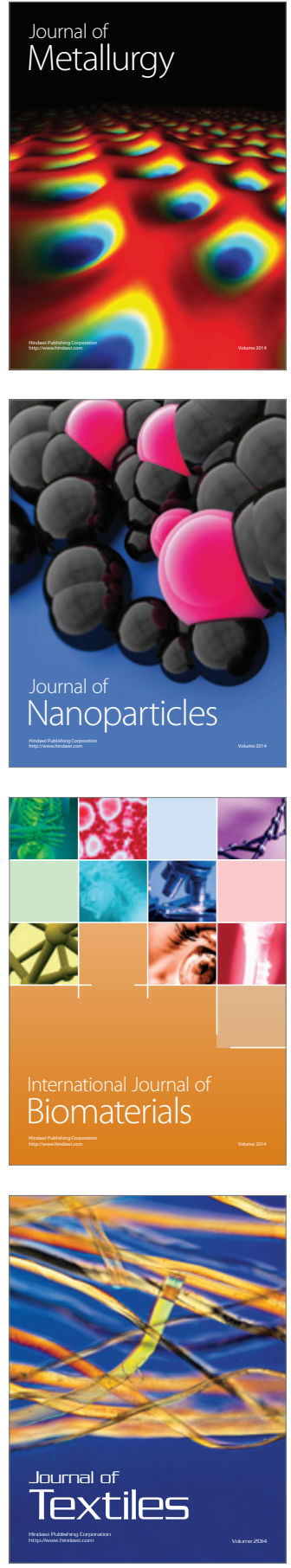